\title{
Primary and post-chemotherapy robotic retroperitoneal lymph node dissection for testicular cancer: a review
}

\author{
Shagnik Ray, Phillip M. Pierorazio, Mohamad E. Allaf \\ Department of Urology, The James Buchanan Brady Urological Institute, Johns Hopkins University School of Medicine, Baltimore, MD, USA \\ Contributions: (I) Conception and Design: All authors; (II) Administrative Support: All authors; (III) Provision of Study Material or Patients: All \\ authors; (IV) Collection and Assembly of Data: All authors; (V) Data Analysis and Interpretation: All authors; (VI) Manuscript Writing: All authors; \\ (VII) Final Approval of Manuscript: All authors. \\ Correspondence to: Mohamad E. Allaf, MD. Department of Urology, The James Buchanan Brady Urological Institute, Johns Hopkins University \\ School of Medicine, Park Building, 600 North Wolfe St. Baltimore, MD 21287, USA. Email: mallaf@jhmi.edu.
}

\begin{abstract}
Open retroperitoneal lymph node dissection (RPLND) is the gold standard for surgical management of the retroperitoneum in patients with testicular cancer, and is associated with excellent oncologic outcomes and significant morbidity including length of stay. Minimally invasive RPLND, starting with laparoscopic retroperitoneal lymph node dissection in 1992 and now robotic retroperitoneal lymph node dissection in 2006, endeavor to decrease the morbidity of open RPLND while maintaining excellent oncologic outcomes. This review surveys the literature regarding both primary and post-chemotherapy robotic RPLND, emphasizing that while early outcomes are promising, much work needs to be done before widespread use of this technique is implemented.
\end{abstract}

Keywords: Robotic; retroperitoneal lymph node dissection; post-chemotherapy; testicular cancer

Submitted Oct 04, 2019. Accepted for publication Jan 21, 2020.

doi: $10.21037 /$ tau.2020.02.09

View this article at: http://dx.doi.org/10.21037/tau.2020.02.09

\section{Introduction}

Accounting for $95 \%$ of testicular cancers, testicular germ cell tumors (GCTs) are the most common solid tumor in men between the ages of 20 and 44 years with an increasing incidence over the past $20+$ years $(1,2)$. Modern treatment paradigms include surgery, platin-based chemotherapy, and radiation therapy and afford high survival rates exceeding $95 \%$ for all men diagnosed with testicular cancer. An important component of this management paradigm is retroperitoneal lymph node dissection (RPLND), an established albeit complex surgical procedure serving as an option in the primary treatment of highrisk clinical stage 1 (CS1) and low-volume clinical stage 2 (CS2) nonseminomatous germ cell tumors (NSGCTs), as well as in the post-chemotherapy setting for residual retroperitoneal masses $>1 \mathrm{~cm}$ in NSGCTs $>3 \mathrm{~cm}$ with PET avidity in men with seminoma (1-3). Recently, RPLND is being investigated in the primary treatment of stage II seminoma (4).

\section{RPLND and alternative management strategies}

Primary PLND performed in an open setting (O-RPLND) is the gold standard for surgical management of the retroperitoneum for men with early-stage (stage I-IIB) NSGCT. When performed at high-volume institutions, $\mathrm{O}-\mathrm{RPLND}$ is associated with excellent oncologic outcomes and low recurrence rates but lengthy hospital stays and significant morbidity including risks of ejaculatory dysfunction, blood loss, ileus, bowel obstruction, chylous ascites, visceral injury, great vessel ligation, and a large surgical scar-all of which can deter patients from pursuing surgery (5). Alternative management strategies for stage I NSGCT include surveillance and adjuvant, platinumbased chemotherapy. While all management strategies for men with Stage I NSGCT are associated with excellent cancer-specific survival rates (approaching 100\%), each management strategy is associated with different exposure rates to chemotherapy (30-100\%) (6). Surveillance is the preferred management option for low risk CS1 disease 
based on the NCCN and AUA guidelines, but any recurrence leads patients to multiple cycles of chemotherapy $(1,7)$. Furthermore, not all patients are able to deal with the uncertainty behind long term surveillance (8). Adjuvant chemotherapy offers a high cure rate with bleomycin, etoposide, and cisplatin, but can results in overtreatment along with significant long-term morbidity including neurotoxicity, ototoxicity, cardiotoxicity, nephrotoxicity, pulmonary toxicity, hypogonadism, infertility, and secondary malignancy (9). Increasing awareness of long-term toxicities associated with chemotherapy has shifted the paradigm from "cure all" to "minimize toxicity" for many patients and providers.

While RPLND may overtreat many patients with stage I NSGCT, it remains an excellent option to minimize exposure to chemotherapy-especially in men with highrisk of relapse (i.e., stage IB). Primary RPLND for stage I NSGCT allows for accurate staging information in conjunction with avoiding the $>20 \%$ relapse rate of patients on surveillance and chemotherapy associated morbidity (8). In a phase III randomized study of RPLND versus primary chemotherapy, $37 \%$ of patients undergoing chemotherapy experienced grade III or IV toxicity relative to only $9 \%$ of patients undergoing RPLND (10). However, patients undergoing RPLND had a recurrence rate of $8 \%$ compared with $0.5 \%$ for chemotherapy. RPLND will overtreatment some patients, but notably $25-35 \%$ of patients who present without radiographic evidence of lymph node pathology will have occult metastasis (11). In men with Stage IIA-B NSGCT, primary RPLND offers a greater than $50 \%$ chance of cure without chemotherapy - an appealing prospect for many patients, especially those with elements of teratoma. Thus, surgeons have sought to improve the morbidity and hospital stays associated with O-RPLND with the advent of minimally invasive RPLND while attempting to carry forward its benefits.

\section{Minimally invasive RPLND}

Initially developed to improve staging of the retroperitoneum, laparoscopic RPLND (L-RPLND) was first performed in 1992 (12). Since 1992, numerous studies demonstrate L-RPLND to have durable longterm oncologic control, nerve sparing capability, less blood loss, shorter post-operative hospital stays, and fewer complications compared with O-RPLND (13). However, L-RPLND is a technically challenging operation and the existing literature represents highly select patient cohorts. In these select cohorts, lower lymph node yield is lower than O-RPLND and oncologic outcomes are obscured by high rates of adjuvant chemotherapy—based on oncologic trends during this time period (13). The first robotic RPNLD (R-RPLND) was performed in 2006 (14). The rationale for the transition was to better replicate open techniques and improve upon the shortcomings created by the technical challenges of pure laparoscopy. Preliminary results demonstrate R-RPLND as non-inferior to O-RPLND and L-RPLND in terms of early oncologic control and safety. R-RPLND has become an important option for CS1 and CS2 NSGCT as well as a feasible strategy for post-chemotherapy retroperitoneal masses. In this article, we discuss the technique behind R-RPLND, its role as primary management of CS1 and CS2 NSGCT and the current status in the post-chemotherapy setting.

\section{Materials and methods}

We performed an electronic PubMed search with the keywords ("robot" or "robotic"), ("retroperitoneal lymph node dissection"), and ("testicular cancer" or "testis cancer") for relevant publications regarding R-RPLND outcomes up to July $1^{\text {st }}, 2019$. Inclusion criteria included single and multi-institutional R-RPLND, O-RPLND, and L-RPLND centered studies for adults with testicular cancer.

\section{Robotic RPLND technique}

R-RPLND is performed using the da Vinci robotic system, with the studies with greater than or equal to 10 patients highlighted in Table 1 utilizing the Si, $\mathrm{X}$, and $\mathrm{Xi}$ system (14-22). Of note, our institution has transitioned from the $\mathrm{Xi}$ system and currently performs R-RPLND with the da Vinci single-port (SP) system, which has facilitated surgery necessitating only a single docking. The surgery is performed via a transperitoneal approach with the patient positioned in either a modified flank position or supine position with the patient placed in Trendelenburg, with the authors' preferred approach being the latter (15-18). In this approach, a Veress needle is used to achieve pneumoperitoneum after which robotic trocars and assistant ports are placed. Of note, bilateral and nerve-sparing dissections can be performed from either method of patient positioning (15-18).

With the $\mathrm{Xi}$ system, the robotic ports are placed in a horizontal and linear fashion at approximately the level of the umbilicus, while port placement for the SP system 


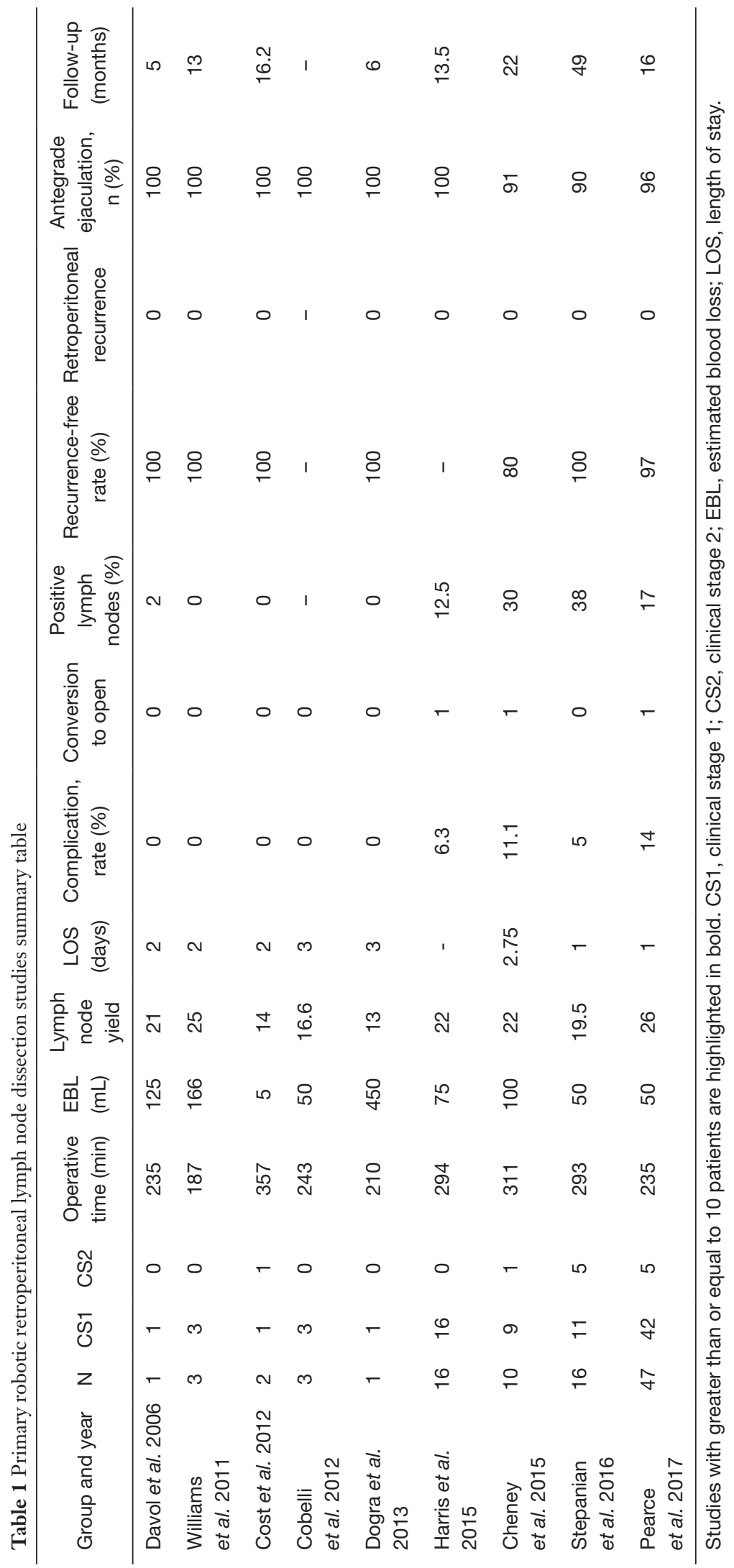


entails a single port placed at the umbilicus. An assistant port is typically placed in the right lower quadrant, with a second assistant port occasionally placed in the contralateral lower quadrant. Much controversy exists regarding the use of templates in early-stage testicular cancer and a full discussion is beyond the scope of this review, with the largest R-RPLND studies showing feasibility of both the unilateral and bilateral templates (15-18). It is the authors' preference to perform a unilateral template for men with CS1 disease and bilateral template for CS2 identified radiographically or operatively. Nerve-sparing is performed in all case where technically feasible.

The robotic approach replicates open RPLND techniques. The ipsilateral colon is reflected to reveal the retroperitoneum, with dissection performed following the boundaries of the iliac bifurcation inferiorly, ureter laterally, and renal vein boundaries superiorly. Once the gonadal vein is identified it is ligated at the level of its origin, with the remainder of the ipsilateral spermatic cord dissected free from the inguinal ring. The left-sided dissection template includes lymph node packets from the left common iliac nodes, preaortic, paraaortic, and retroaortic regions. The right-sided dissection template includes lymph nodes from the paracaval, interaortocaval, and preaortic spaces. During dissection the sympathetic chain and postganglionic nerve fibers are identified and preserved. To prevent postoperative lymphatic leak, a combination of electrocautery and hem-olok clips are used to clamp lymph node packets. To facilitate retrocaval and retroaortic lymph node packets, control of lumbar vessels via ligation is performed with surgical clips, ties, or suture ligation.

The robotic system's wristed instruments allow for improved dexterity to allow for dissection of all retrocaval and retroaortic tissue. Furthermore, nerve dissection and preservation are made easier by the magnified camera view and camera angle. Once hemostasis is achieved, fibrin sealant can be applied to lymphatic beds to help prevent lymphatic leaks. We do not typically place a drain at our center.

After recovery in a post-acute care unit, patients are transferred to the floor. Diet is quickly advanced from clear liquids (night of surgery) to fat-free regular diet (day after surgery). A low-fat diet is initiated in the hospital and accompanied by a nutrition consult. The fat content is regularly advanced over 4 weeks to decrease the risk of lymphatic leak. Patients are discharged when they are ambulatory, tolerating a regular diet and pain is wellcontrolled; typically post-operative day one or two. We encourage patients to remain out of school or work for two weeks and to avoid strenuous physical activity for three to four weeks.

\section{Primary R-RPLND}

To date, there are multiple works published detailing the outcomes and complications of primary R-RPLND (23). We highlight the four largest series so far in Table 1. Of note, all four studies take place at high-volume academic centers with experienced robotic surgeons and are retrospective in design, and to date no completely prospective trial regarding R-RPLND has been performed to the best of our knowledge.

R-RPLND as a minimally invasive urologic surgery is expected to have longer operative time but less blood loss, shorter recovery time, and decreased hospital stay than its open counterpart. R-RPLND across the four studies had median operative times ranging from 235-311 minutes, much greater than compared with the median operative times in a large metanalysis of both L-RPLND (204 minutes) and O-RPLND (186 minutes) (13,15-18). Median blood loss across the four studies ranged from 50$100 \mathrm{~mL}$, much lower than the range of $150-325 \mathrm{~mL}$ found in different open series (24-27). When compared with the laparoscopic approach, Harris et al. found similar blood loss with R-RPLND (75 mL; IQR, 50-100 mL) and L-RPLND (125 mL; IQR, 50-150 mL) (15). Like with other minimally invasive surgeries involving insufflation, this decreased blood loss is likely secondary to the increased abdominal pressure controlling venous bleeding. Median length of stay ranged from 1-3 days for R-RPLND, shorter than that reported for both L-RPLND (3.3 days) and O-RPLND (6.6 days) (13). This is likely secondary to a smaller incision and decreased postoperative ileus, resulting in patients with improved pain control who are able to get out of bed and walk earlier and regain bowel function. Notably, conversion to open was an uncommon occurrence (3.4\% of all patients in the four studies), which is similar to that noted for L-RPLND (3.7\%) (13).

One of the most glaring drawbacks to O-RPLND is its relatively high rate of complications $(5 \%, 24 \%$, and $7 \%$ for intraoperative, postoperative, and late complications respectively in one more recent series) (5). Recognizing the selection of favorable cases in the early adoption of R-RPLND, the reported major complication rates are low. Harris et al. reported 1 (6.2\%) intraoperative complication, an aortic injury necessitating open conversion with 
successful open repair of the injury (15). Stepanian et al. reported having $1(6.25 \%)$ intraoperative complication in their series consisting of a transected ureter that was subsequently repaired with success (16). Pearce et al. reports $2(4.3 \%)$ intraoperative complications, a pancreatic injury that was recognized intraoperatively and managed with drain placement, and an aortic injury necessitating open conversion and subsequent successful repair (18). The rate of intraoperative complication was thus similar to that seen in open series, albeit difficult to properly compare given the low sample size of R-RPLND.

The postoperative complication rate likewise is comparable to that of O-RPLND and L-RPLND. Harris et al. reported $1(6.2 \%)$ postoperative complication (Clavien III) (15). Cheney et al. noted having only 1 (10\%) postoperative complication (ileus) (17). Stepanian et al. report only 2 patients with retrograde ejaculation as postoperative complications (16). Finally, Pearce et al. reported having 4 (8.5\%) postoperative complications (18). Two were Clavien grade I (chylous ascites resolving with medium chain triglyceride diet, ileus requiring nasogastric tube decompression) and 2 were Clavien Grade III (body wall hematoma requiring 1 unit of packed red blood cells, chylous ascites requiring a single course of paracentesis) (18). Pearce et al.'s complication rate of $8.5 \%$ is comparable to the complication rate of L-RPLND in a metanalysis (15.5\%) and O-RPLND in more recent series $(7-24 \%)(5,26)$. While not reported in any of the other highlighted studies, $4.3 \%$ (2) patients in the Pearce et al. series experienced chylous ascites, with one patient requiring paracentesis. This high rate is surprising, as one would expect the improvements in visualization and dexterity associated with robotic surgery to allow for improved ligation of lymphatics. It is unclear if this high rate is secondary to small sample size, but suggests that surgeons must be aware and able to manage this complication in R-RPLND patients. The insufflation pressures during laparoscopy may obscure small lymphatic leaks leading to a slightly higher rate in the robotic experience. Subramanian et al. reported that $18 \%$ of patients undergoing O-RPLND experienced postoperative ileus compared with only $2 \%$ of patients undergoing R-RPLND in Pearce et al.'s cohort $(5,18)$. Given the significant economic burden of postoperative ileus studied in other types of surgeries, this is a significant benefit of the robotic approach, likely secondary to differences in bowel mobilization, less morbid incision with improved pain control, and quicker return to ambulation. Notably, all series reported excellent reports of antegrade ejaculation, ranging from $90-100 \%$, with Pearce et al. noting an antegrade ejaculation rate of $96 \%$ (15-18). Potential longterm sexual dysfunction can cause significant morbidity for testicular cancer survivors, with these results indicating that R-RPLND can replicate nerve-sparing procedures.

Early oncologic outcomes are favorable in the reported literature. The lymph node yield, which can represent the extent of node dissection, ranged from 19.5 to 26 across the four studies-slightly lower relative to O-RPLND (ranging from $28-38$ in different studies) $(28,29)$. In spite of this, recurrence rates fared well and ranged from $80 \%$ to $100 \%$, with the largest study comprising 47 patients (which includes our institution's patient cohort) by Pearce et al. having a recurrence free rate of $97 \%$ over a median follow-up time of 16 months (18). In the Pearce et al. cohort, 42 patients $(89.4 \%)$ were CS1 while the remaining 5 (10.6\%) were CS2. Of the 8 patients (17\%) with positive lymph nodes, 5 underwent adjuvant chemotherapy. No patients experienced recurrence in the Stepanian et al. cohort comprising $11 \mathrm{CS} 1$ patients and 5 CS2 patients (16). Thirty-eight percent $(\mathrm{n}=6)$ patients in that primary RPLND cohort had positive lymph nodes, with 2 patients receiving adjuvant chemotherapy. 8 patients (32\%) from this study had positive lymph nodes on pathology, with only 2 out of 8 patients received adjuvant therapy secondary to embryonal carcinoma noted on final pathology. Of the remaining 6 patients with positive lymph nodes, three were positive for teratoma and thus did not need any adjuvant therapy. Of the recurrences in all four early outcome studies, none were retroperitoneal in nature (15-18).

These early outcomes are promising and comparable to the recurrence-free rates of O-RPLND (92.5\%) and L-RPLND (95.4\%), with no reported retroperitoneal recurrences (13). Unfortunately, comparing open, laparoscopic and robotic series is challenging given these studies use slightly different surgical techniques, vary in utilization of adjuvant chemotherapy, and have relatively short follow-up time. Accruing data from these series will determine the oncologic efficacy of R-RPLND. Recent literature indicates oncologic concerns with R-RPLND. Calaway et al. present a case series of 5 patients who underwent R-RPLND with atypical patterns of recurrence for patients with low risk NSGCT (30). In the series, four patients presented with low-risk NSGCT and 1 with intermediate-risk NSGCT; three underwent primary R-RPLND and two underwent post-chemotherapy R-RPLND. Recurrences were discovered at a median time of 259 days (92-503 days) with one in-field recurrence 
and four out-of-field recurrences (pericolic space with invasion into the colon, peritoneal carcinomatosis in addition to a perinephric mass, large volume liver lesions with suprahilar disease extending in the retrocrural space, and lymph nodes in the celiac axis). All patients underwent additional chemotherapy and three patients required additional surgery. One patient died of disease. This report casts a concerning shadow over the oncologic outcomes of R-RPLND. It is challenging to discern the impact of robotic technology on oncologic outcomes when the denominator of recurrences is unknown, surgical technique, and surgeon experience are unknown. With more data, the efficacy of R-RPLND will be determined. Given the excellent oncologic outcomes of O-RPLND, any deviation from the standard of care requires careful study before widespread adoption at less experienced centers. Therefore, we recommend R-RPLND only be performed by surgeons with significant experience in testicular cancer, RPLND and robotic surgery—a stance endorsed by American Urological Association Guidelines (7).

\section{Post-chemotherapy R-RPLND}

Postchemotherapy RPLND (PC-RPLND) presents several challenges not present for primary RPLND and that make the role of R-RPLND more dubious. PCRPLND is notable for high complication rates and concomitant surgery (including vascular repair, bowel resections, nephrectomy, etc.) given the desmoplastic reaction induced by chemotherapy and subsequent merging of tissue planes complicating tissue dissection $(31,32)$. Importantly, oncologic outcomes following PC-RPLND are dependent on quality surgery as options for salvage are limited (33). PC-RPLND is not performed robotically at our center given the concerns expressed above, nevertheless a number of centers report preliminary outcomes regarding postchemotherapy robotic RPLND (PC-R-RPLND) in small series. The studies reporting outcomes for PC-RRPLND are summarized in Table 2 (16,17,34-40).

Cheney et al. compared eight men undergoing PCR-RPLND with ten men who underwent primary RPLND (17). There was no retroperitoneal recurrence at a median follow up of 22 months. There was no difference in lymph node yield (primary 22, postchemotherapy 18), estimated blood loss (primary 100, postchemotherapy 313), and length of hospital stay (primary 2.75 days, postchemotherapy 2.2 days). However, operative time for PC-R-RPLND (369 minutes) was significantly longer than for primary R-RPLND (311 minutes) and two men undergoing PC-R-RPLND (22.3\%) were converted to open surgery.

Kamel et al. retrospectively reported 12 patients undergoing PC-R-RPLND. One patient required open conversion, two (18.2\%) minor complications (postoperative ileus and upper limb neuropathy) and one (9.1\%) major complication (aortic injury) were reported (34). Singh et al. reported 13 patients with clinical stage II or higher disease who underwent postchemotherapy R-RPLND. Three patients $(23.1 \%)$ had teratoma in their lymph nodes. Over a median of 23 months (range, 3-58 months), there were no systemic or retroperitoneal recurrences (35). Ten complications were reported including one intraoperative aortic injury, four $(30.8 \%)$ chyle leaks, and five patients with post-operative ileus. Additionally, Overs et al. reported on 11 patients who underwent postchemotherapy R-RPLND, ten with CS2 and one with CS3 (36). They reported no intraoperative complications, and reported only 1 (9.1\%) postoperative complication (chylous ascites). $72.7 \%$ of their patients reported normal, antegrade ejaculation 1 month after surgery. At a median 2 years from surgery, no patients had recurrence. Lymph node yield in this series was low (median 7) and attributed to utilization of modified unilateral lymph node templates.

Most recently, in the largest series to date, Li et al. retrospectively compared 30 patients who underwent R-PC-RPLND with 63 patients who underwent open PCRPLND (O-PC-RPLND) (38). Baseline clinicopathologic patient characteristic differences between R-PC-PRLND and O-PC-RPLND reflected careful selection for robotic surgery and included lower clinical stage $(\mathrm{P}=0.006)$, favorable International Germ Cell Cancer Collaborative Group classification $(\mathrm{P}=0.01)$, marked response to chemotherapy $(\mathrm{P}=0.002)$, and smaller retroperitoneal mass postchemotherapy $(\mathrm{P}=0.001)$ (38). O-PC-RPLND (375 minutes) and R-PC-RPLND (388 minutes) had similar operative time, with significantly more EBL in the O-PCRPLND group (825 vs. $235 \mathrm{~mL}$; $\mathrm{P}<0.001$ ). Similar to primary RPLND, the median length of stay for O-PCRPLND was greater than that of R-PC-RPLND (7 vs. 2 days; $\mathrm{P}<0.001)$. The complication rate was high $(33 \%)$, and the difference between the R-PC-RPLND (20\%) and O-PC-RPLND (39.7\%) group did not reach statistical significance, potentially due to the effects of small sample size. Three patients required conversion to open surgery for a failure to progress secondary to inadequate visualization $(n=2)$ and inability to control a vascular injury $(n=1)$. The 


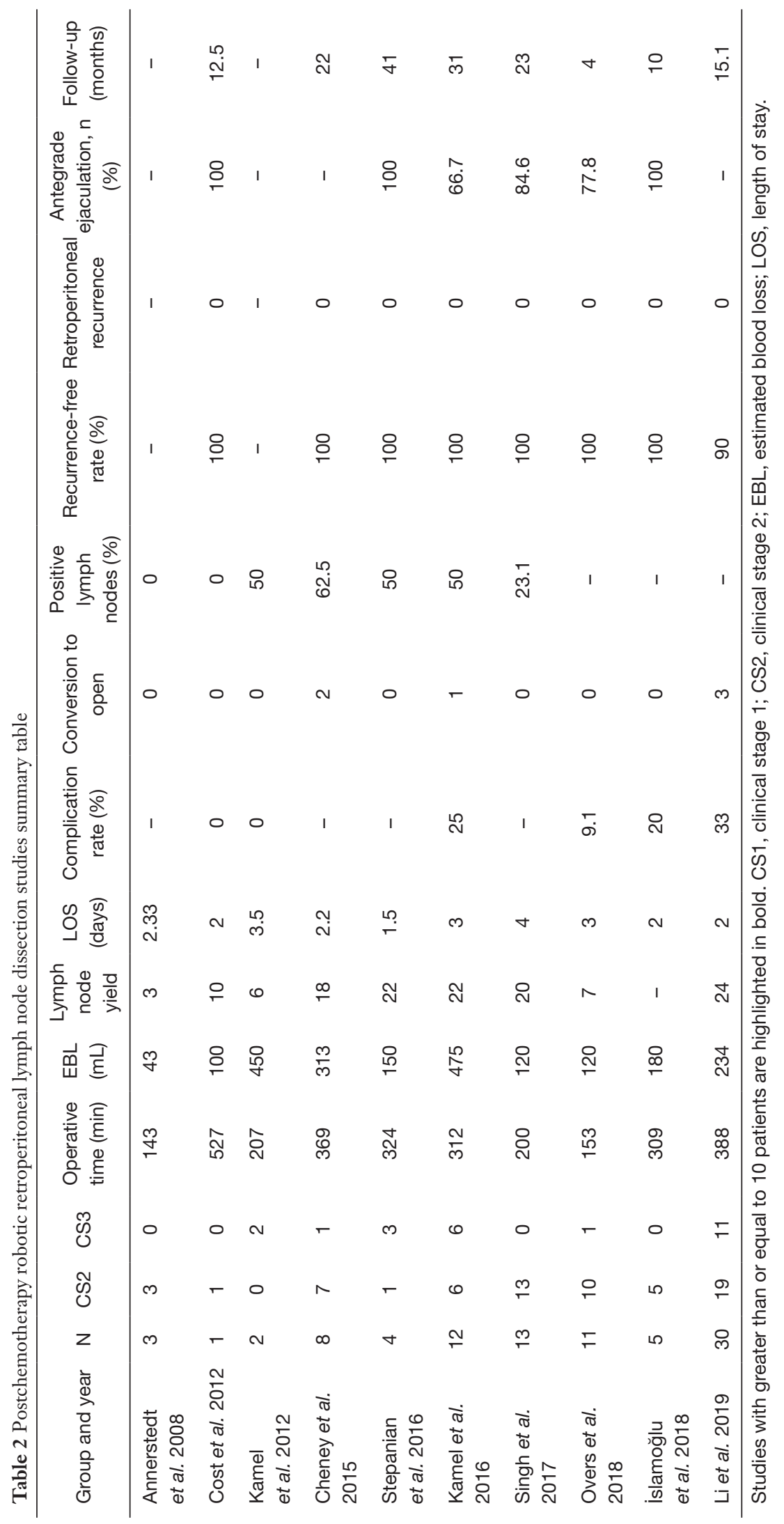


major complications after R-PC-RPLND included chylous ascites $(n=1)$, pneumothorax $(n=1)$, and colon perforation $(\mathrm{n}=1)$. Oncologic outcomes were similar between groups, with the authors not finding that surgical approach significantly predicted time to recurrence for these patients. Two patients $(6.7 \%)$ required additional procedures by consulting surgical services for maximal oncologic control.

The existing literature regarding PC-R-RPLND is most notable for high rates of chylous ascites and short oncologic follow-up. Furthermore, in order to achieve maximal oncologic control, ancillary surgical procedures have been shown to be required in the O-PC-RPLND literature and were noted as necessary by $\mathrm{Li}$ et al. for R-PC-RPLND $(32,38)$. This could necessitate conversion to open surgery given the consulting surgeon's familiarity with robotic surgery or degree of emergency, a risk that can theoretically be minimized by excluding patients from R-PC-RPLND who are at higher risk of requiring adjuvant surgical intervention (e.g., retroperitoneal mass $>5 \mathrm{~cm}$, intermediate or poor IGCCCG classification) (32). As stated above, we do not routinely perform R-RPLND in the postchemotherapy setting and recommend that these surgeries only be performed in select settings by experts in testicular cancer, RPLND, and robotic surgery. Accruing data from larger and more robust prospective studies are required before R-RPLND can be considered routine in the postchemotherapy setting.

\section{Conclusions}

R-RPLND is a minimally invasive approach to earlystage testicular cancer that endeavors to reduce the burden of long-term toxicities from chemotherapy and the considerable morbidity of O-RPLND while maintaining oncologic control. Early results from high-volume, experienced academic centers demonstrate the feasibility of R-RPLND with strong early oncologic outcomes and appropriate complication rates. Larger, prospective studies with longer follow-up are necessary to better evaluate oncologic outcomes and concerns regarding patterns of recurrence. PC-R-RPLND is supported by a few small studies with limited follow-up with reports of significant morbidity (chyle leak, postoperative ileus), suggesting that this approach needs further study before widespread adoption. Given the significant technical complexities of patient selection and the operation, R-RPLND should only be performed by surgeons with expertise in testicular cancer, RPLND and robotic surgery.

\section{Acknowledgments}

Funding: None.

\section{Footnote}

Provenance and Peer Review: This article was commissioned by the Guest Editor (Ashok K. Hemal) for the series "Robotic-assisted Urologic Surgery" published in Translational Andrology and Urology. The article was sent for external peer review organized by the Guest Editor and the editorial office.

Conflicts of Interest: The series "Robotic-assisted Urologic Surgery" was commissioned by the editorial office without any funding or sponsorship. The authors have no other conflicts of interest to declare.

Ethical Statement: The authors are accountable for all aspects of the work in ensuring that questions related to the accuracy or integrity of any part of the work are appropriately investigated and resolved.

Open Access Statement: This is an Open Access article distributed in accordance with the Creative Commons Attribution-NonCommercial-NoDerivs 4.0 International License (CC BY-NC-ND 4.0), which permits the noncommercial replication and distribution of the article with the strict proviso that no changes or edits are made and the original work is properly cited (including links to both the formal publication through the relevant DOI and the license). See: https://creativecommons.org/licenses/by-nc$\mathrm{nd} / 4.0 /$.

\section{References}

1. Motzer RJ, Jonasch E, Agarwal N, et al. Testicular Cancer, Version 2.2015. J Natl Compr Canc Netw 2015;13:772-99.

2. Albers $\mathrm{P}$, Albrecht W, Algaba F, et al. Guidelines on Testicular Cancer: 2015 Update. Eur Urol 2015;68:1054-68.

3. Patel HD, Joice GA, Schwen ZR, et al. Retroperitoneal lymph node dissection for testicular seminomas: population-based practice and survival outcomes. World $\mathrm{J}$ Urol 2018;36:73-8.

4. Pierorazio PM, Biles MJ. Indications for Surgery in Disseminated Seminoma. Urol Clin North Am 
2019;46:399-407.

5. Subramanian VS, Nguyen CT, Stephenson AJ, et al. Complications of open primary and post-chemotherapy retroperitoneal lymph node dissection for testicular cancer. Urol Oncol 2010;28:504-9.

6. Hamilton RJ, Nayan M, Anson-Cartwright L, et al. Treatment of Relapse of Clinical Stage I

Nonseminomatous Germ Cell Tumors on Surveillance. J Clin Oncol 2019;37:1919-26.

7. Stephenson A, Scott E, Eric B, et al. Diagnosis and Treatment of Early Stage Testicular Cancer: AUA Guideline. J Urol 2019;202:272-81.

8. Kollmannsberger C, Tandstad T, Bedard PL, et al. Patterns of relapse in patients with clinical stage I testicular cancer managed with active surveillance. J Clin Oncol 2015;33:51-7.

9. Chovanec M, Abu Zaid M, Hanna N, et al. Long-term toxicity of cisplatin in germ-cell tumor survivors. Ann Oncol 2017;28:2670-9.

10. Albers P, Siener R, Krege S, et al. Randomized phase III trial comparing retroperitoneal lymph node dissection with one course of bleomycin and etoposide plus cisplatin chemotherapy in the adjuvant treatment of clinical stage I Nonseminomatous testicular germ cell tumors: AUO trial AH 01/94 by the German Testicular Cancer Study Group. J Clin Oncol 2008;26:2966-72.

11. Fernandez EB, Moul JW, Foley JP, et al. Retroperitoneal imaging with third and fourth generation computed axial tomography in clinical stage I nonseminomatous germ cell tumors. Urology 1994;44:548-52.

12. Rukstalis DB, Chodak GW. Laparoscopic retroperitoneal lymph node dissection in a patient with stage 1 testicular carcinoma. J Urol 1992;148:1907-9; discussion 1909-10.

13. Rassweiler JJ, Scheitlin W, Heidenreich A, et al. Laparoscopic retroperitoneal lymph node dissection: does it still have a role in the management of clinical stage I nonseminomatous testis cancer? A European perspective. Eur Urol 2008;54:1004-15.

14. Davol P, Sumfest J, Rukstalis D. Robotic-assisted laparoscopic retroperitoneal lymph node dissection. Urology 2006;67:199.

15. Harris KT, Gorin MA, Ball MW, et al. A comparative analysis of robotic vs laparoscopic retroperitoneal lymph node dissection for testicular cancer. BJU Int 2015;116:920-3.

16. Stepanian S, Patel M, Porter J. Robot-assisted Laparoscopic Retroperitoneal Lymph Node Dissection for Testicular Cancer: Evolution of the Technique. Eur Urol
2016;70:661-7.

17. Cheney SM, Andrews PE, Leibovich BC, et al. Robotassisted retroperitoneal lymph node dissection: technique and initial case series of 18 patients. BJU Int 2015;115:114-20.

18. Pearce SM, Golan S, Gorin MA, et al. Safety and Early Oncologic Effectiveness of Primary Robotic Retroperitoneal Lymph Node Dissection for Nonseminomatous Germ Cell Testicular Cancer. Eur Urol 2017;71:476-82.

19. Williams SB, Lau CS, Josephson DY. Initial Series of Robot-Assisted Laparoscopic Retroperitoneal Lymph Node Dissection for Clinical Stage I Nonseminomatous Germ Cell Testicular Cancer. Eur Urol 2011;60:1299-302.

20. Cost NG, DaJusta DG, Granberg CF, et al. Robot-Assisted Laparoscopic Retroperitoneal Lymph Node Dissection in an Adolescent Population. J Endourol 2012;26:635-40.

21. Dogra PN, Singh P, Saini AK, et al. Robot assisted laparoscopic retroperitoneal lymph node dissection in testicular tumor. Urol Ann 2013;5:223.

22. de Cobelli O, Brescia A, Mazzoleni F, et al. A novel "intuitive" surgical technique for right robot-assisted retroperitoneal lymph node dissection for stage I testicular NSGCT. World J Urol 2013;31:435-9.

23. Tselos A, Moris D, Tsilimigras DI, et al. Robot-Assisted Retroperitoneal Lymphadenectomy in Testicular Cancer Treatment: A Systematic Review. J Laparoendosc Adv Surg Tech A 2018;28:682-9.

24. Syan-Bhanvadia S, Bazargani ST, Clifford TG, et al. Midline Extraperitoneal Approach to Retroperitoneal Lymph Node Dissection in Testicular Cancer: Minimizing Surgical Morbidity. Eur Urol 2017;72:814-20.

25. Beck SDW, Foster RS, Bihrle R, et al. Is full bilateral retroperitoneal lymph node dissection always necessary for postchemotherapy residual tumor? Cancer 2007;110:1235-40.

26. Williams SB, McDermott DW, Winston D, et al. Morbidity of open retroperitoneal lymph node dissection for testicular cancer: contemporary perioperative data. BJU Int 2010;105:918-21.

27. Heidenreich A, Pfister D. Retroperitoneal lymphadenectomy and resection for testicular cancer: an update on best practice. Ther Adv Urol 2012;4:187-205.

28. Nayan M, Jewett MAS, Sweet J, et al. Lymph Node Yield in Primary Retroperitoneal Lymph Node Dissection for Nonseminoma Germ Cell Tumors. J Urol 2015;194:386-91.

29. Thompson RH, Carver BS, Bosl GJ, et al. Evaluation of 
lymph node counts in primary retroperitoneal lymph node dissection. Cancer 2010;116:5243-50.

30. Calaway AC, Einhorn LH, Masterson TA, et al. Adverse Surgical Outcomes Associated with Robotic Retroperitoneal Lymph Node Dissection Among Patients with Testicular Cancer. Eur Urol 2019;76:607-9.

31. Cary C, Masterson TA, Bihrle R, et al. Contemporary trends in postchemotherapy retroperitoneal lymph node dissection: Additional procedures and perioperative complications. Urol Oncol 2015;33:389.e15-21.

32. Djaladat H, Nichols C, Daneshmand S. Adjuvant surgery in testicular cancer patients undergoing postchemotherapy retroperitoneal lymph node dissection. Ann Surg Oncol 2012;19:2388-93.

33. Sharp DS, Carver BS, Eggener SE, et al. Clinical outcome and predictors of survival in late relapse of germ cell tumor. J Clin Oncol 2008;26:5524-9.

34. Kamel MH, Littlejohn N, Cox M, et al. PostChemotherapy Robotic Retroperitoneal Lymph Node Dissection: Institutional Experience. J Endourol 2016;30:510-9.

35. Singh A, Chatterjee S, Bansal P, et al. Robot-assisted retroperitoneal lymph node dissection: Feasibility and

Cite this article as: Ray S, Pierorazio PM, Allaf ME. Primary and post-chemotherapy robotic retroperitoneal lymph node dissection for testicular cancer: a review. Transl Androl Urol 2020;9(2):949-958. doi: 10.21037/tau.2020.02.09 outcome in postchemotherapy residual mass in testicular cancer. Indian J Urol 2017;33:304-9.

36. Overs C, Beauval JB, Mourey L, et al. Robot-assisted post-chemotherapy retroperitoneal lymph node dissection in germ cell tumor: is the single-docking with lateral approach relevant? World J Urol 2018;36:655-61.

37. İslamoğlu E, Özsoy Ç, Anıl H, et al. Post-chemotherapy robot-assisted retroperitoneal lymph node dissection in non-seminomatous germ cell tumor of testis: Feasibility and outcomes of initial cases. Turk J Urol 2018;45:113-7.

38. Li R, Duplisea JJ, Petros FG, et al. Robotic Postchemotherapy Retroperitoneal Lymph Node Dissection for Testicular Cancer. Eur Urol Oncol 2019. [Epub ahead of print].

39. Kamel MH, Jackson CM, Moore JT, et al. Postchemotherapy robotic retroperitoneal lymph node dissection (RRPLND) in testicular cancer. J Robot Surg 2012;6:359-62.

40. Annerstedt M, Gudjonsson S, Wullt B, et al. Robotassisted laparoscopic retroperitoneal lymph node dissection in clinical stage II testicular cancer. J Robot Surg 2008;2:189-91. 